Document downloaded from:

http://hdl.handle.net/10251/160217

This paper must be cited as:

Valero Chuliá, FJ.; Rubio Montoya, FJ.; Llopis Albert, C. (2019). Assessment of the Effect of Energy Consumption on Trajectory Improvement for a Car-like Robot. Robotica. 37(11):1998-2009. https://doi.org/10.1017/S0263574719000407

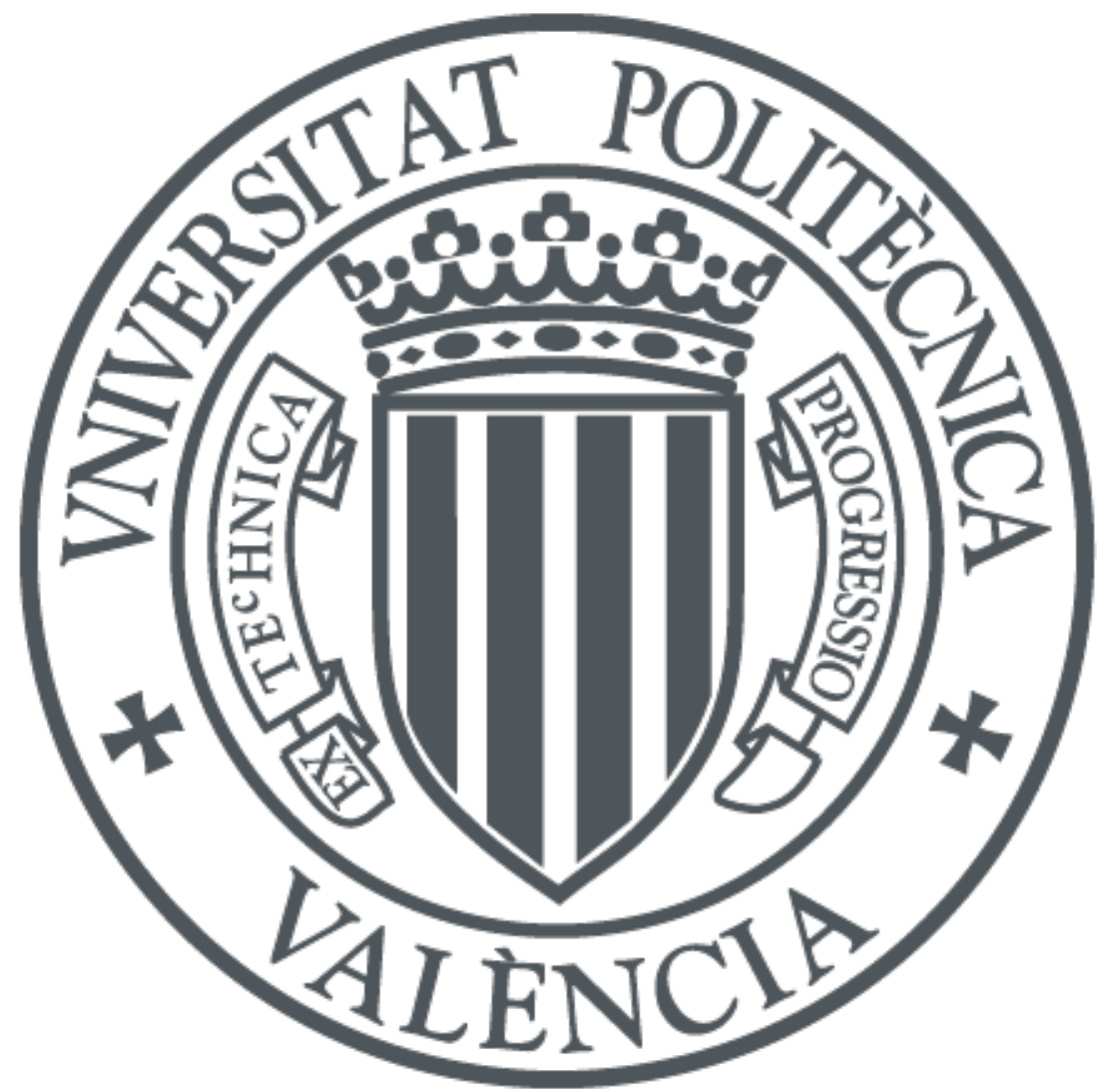

The final publication is available at

https://doi.org/10.1017/S0263574719000407

Copyright Cambridge University Press

Additional Information 


\title{
Assessment of the effect of energy consumption on trajectory improvement for a car-like robot
}

\author{
Francisco Valero ${ }^{1}$; Francisco Rubio ${ }^{1}$; Carlos Llopis-Albert ${ }^{2}$ \\ ${ }^{1}$ Center of Technological Research in Mechanical Engineering, Building 5E, Universitat Politècnica de València, \\ Spain. \\ E-mail: fvalero@mcm.upv.es; Corresponding author: frubio@mcm.upv.es; \\ ${ }^{2}$ Department of Mechanical Engineering and M. Strength, Universitat Politècnica de València, Spain. E-mail: \\ cllopisa@upvnet.upv.es
}

\begin{abstract}
Reducing the energy consumed by a car-like mobile robot makes it possible to move at a lower cost, yet it takes more working time. This paper proposes an optimization algorithm for trajectories with optimal times and analyzes the consequences of restricting the energy consumed on the trajectory obtained for a car-like robot. When modeling the dynamic behavior of the vehicle, it is necessary to consider its inertial parameters, the behavior of the motor and the basic properties of the tire in its interaction with the ground. To obtain collision-free, minimum-time trajectories quadratic sequential optimization techniques are used, where the objective function is the time taken by the robot to move between two given configurations. This is subject to constraints relating to the vehicle and tires as well as the energy consumed, which is the basis for this paper. We work with a real random distribution of consumed energy values following a normal Gaussian distribution in order to analyze its influence on the trajectories obtained by the vehicle. The energy consumed, the time taken, the maximum velocity reached and the distance traveled are analyzed in order to characterize the properties of the trajectories obtained. The proposed algorithm has been applied to 101 examples, showing that the computational times needed to obtain the solutions are always lower than those required to realize the trajectories. The results obtained allow us to reach conclusions about the energy efficiency of the trajectories.
\end{abstract}

Keywords: Energy consumed, car-like mobile robot, robot dynamics, tire interaction, minimumtime trajectory, collision-free trajectory, working parameters.

\section{Introduction}

Autonomous vehicles, or car-like robots, are transforming the way we conceive transport. Their use has increased notably in recent years due to advances in sensor and control systems. These vehicles range from the Google car to the modern AGV (automated guided vehicle) used in industrial applications to move materials in manufacturing, value-added and storage operations. The most important advantages of autonomous vehicles are related to the safety of driving, efficiency in energy consumption, the fluidity of transport, the release of users or operators from the monotonous task of driving and the enormous potential for use by all types of people, applications and companies.

In obtaining the motion to be followed by the autonomous vehicle, it is very useful to generate optimal trajectories. This is a field that is still open and has traditionally been very fertile, where 
researchers have made great contributions. One of the most recent works on this particular matter appears in [1], where a trajectory generation algorithm that is especially well suited to wheeled mobile robots (WMRs) is presented. Linear and angular velocities of the WMR are specified as Bézier polynomials. The algorithm helps to avoid slipping and skidding of the WMR, since smooth starting and braking can be achieved by proposing suitable linear and angular velocities. In [2] the state of the art and the latest studies on motion planning for autonomous robots are detailed. A compilation of the most important contributions during recent decades from a different point of view can be seen in [3]. In [4] a review of motion planning is provided, focusing specifically on autonomous vehicles, with a comparison between some of the techniques used.

Another aspect that needs to be considered in motion planning for car-like robots is their dynamic model, including characterization of the tire's interaction with the terrain. Dynamic modeling of the robot is fundamental in order to determine whether or not the vehicle will be able to describe the calculated trajectories. In addition, it brings a degree of realism to the analysis of the vehicle's behavior that increases the more accurate the model is. On the other hand, the forces between tire and terrain have a great effect on the efficiency with which the trajectory is carried out.

A study that makes use of dynamic modeling of the robot and its interaction with the terrain can be seen in [5]. The author models forces, but does not delve into the nature of them, oversimplifying the way they are obtained.

In [6] the authors analyze the problem of finding optimal trajectories for autonomous vehicles considering the dynamic model of the vehicle, but treating its behavior as a particle and thus losing generality for the analysis of specific robots that do not admit such simplification.

An important part of dynamic modeling is the interaction forces between the tire and the terrain. In [7] a comprehensive analysis is made of these forces for planetary rovers. This paper discusses the significance of the study of wheel-soil interaction mechanics of planetary rovers and summarizes the differences between planetary rovers and terrestrial vehicles.

A more recent paper, which analyzes the influence of friction forces on the execution of optimal trajectories, can be seen in [8]. In this paper a dynamic model of the car-like robot is used, in which the driving, friction forces and tire-ground interactions are clearly distinguished.

An improvement in the motion optimization of car-like robots comes from considering the energy that will be consumed by the robot to perform the planned trajectories. The relationship between the energy available and the energy consumed is fundamental for the autonomous vehicle to work, since the vehicle has a limited amount of energy and this approach seeks to reduce consumption. A lower consumption means increasing the working time. In addition, the more efficient and the lower the energy consumption, the more economical driving will be. Obviously, it is necessary to optimize the amount of energy that an autonomous vehicle will use to carry out operations.

In [9] the problem of minimum-energy trajectory planning for differentially-driven car-like robots was addressed. This paper proposes the minimum-energy rotational trajectory for a wheeled mobile robot (WMR) that minimizes the energy drawn from the batteries. Optimal control theory is used to achieve the energy-optimal velocity profiles.

In [10], the problem of finding optimal trajectories that minimize the energy consumed during motion of a car-like robot on flat surfaces is analyzed. They separate the problem into two parts, trying to obtain optimal energy velocities profiles and optimal energy path profiles. A very important drawback of this work is that it does not take into account the dynamic characteristics 
of the car-like robot. They try to optimize the energy simply by improving the acceleration and deceleration profiles of the DC electric motor used by the robot based on simplifications of the forces acting on the robot. They also fail to consider the existence of potential obstacles that could collide with the robot.

A cost function that weights force inputs, area covered by an unmanned ground vehicle (UGV) and motor efficiency to create an optimal trajectory is presented in [11]. The trajectory is constrained to follow a described path. The trade-offs are discussed (including the time required to cover the region and the energy required to complete the trajectory). The authors over-simplify the dynamics of the robot by considering it as a point. Also, when seeking a path that optimizes the energy consumption, the algorithm becomes more and more complex. The dynamics come from the Wong's model in [12]. The algorithm is very costly. This paper builds on previous work carried out by the authors in [13].

In [14] the authors propose the minimization of the energy expended by a car-like robot when moving along a road with a known profile. The constraints are given by means of a simple model of the longitudinal dynamics.

In [15] a Model Predictive Control (MPC) strategy for energy-efficient motion control of car-like vehicles is presented. The resulting strategy drives the vehicle along energy-efficient trajectories around the desired one. The distance between the closed-loop trajectories and the desired trajectory provided by the user is guaranteed to be ultimately bounded. Numerical results show the effectiveness of the proposed control strategy for the case of a car driven over flat land or mountainous terrain. The authors work with the bicycle model of the car-like robot. In [16] a new method of optimal motion planning is presented, which aims to minimize the energy consumption of a wheeled mobile robot in robot applications. First a model that can be used to formulate the energy consumption for kinetic energy transformation and to overcome traction resistance is developed. This model will provide a base for minimizing the robot's energy consumption through proper motion planning. An $\mathrm{A}^{*}$ algorithm is employed to generate an energy-efficient path where a new energy-related criterion is utilized in the cost function. To achieve a smooth trajectory along the generated path, the appropriate arrival time and velocity at the defined waypoints are selected for minimum energy consumption. Simulations and experiments are performed to demonstrate the energy-saving efficiency of the proposed motion planning algorithm.

A recent work that also takes into account the energy consumed by a robot can be found in [21], although it does not apply to a car-like robot. In it, the authors present an optimal trajectory planning method for industrial robots focusing on the least time and energy consumption without violating the kinematic constraints.

This paper introduces an optimal trajectory planning algorithm for car-like robots. It takes into account the robot's dynamic properties and works efficiently in terms of computational time. It is based on solving optimization problems and focuses on how the energy consumed influences trajectory planning and affects working parameters such as minimum-time trajectory, maximum speed of the robot and computational time.

To carry out this study energy values are taken using the Montecarlo method from a normal Gaussian distribution that tries to reproduce real values about the robot's energy.

The dynamic approach includes modeling the whole car-like robot (engine and transmission system, tires and brakes) with the aim of calculating feasible and efficient trajectories for the robot 
and to analyze how the energy values affect the robot's performance. This approach is different to other planners that only include kinematic constraints, as in Simba et al. [17], or that do not guarantee the feasibility of the trajectories, as in [10].

Efficient computation time is achieved by simplifying the dynamic model, rapidly evaluating collisions, as explained in [18], and generating a trajectory that is subject to energy constraints. The efficiency of the trajectory comes from the full dynamic capability of the robot and it is shown in the examples analyzed. A large number of examples (101) have been solved, varying the available energy. The results have been plotted in section 5 .

\section{The robotic vehicle}

The RBK robot is an electric vehicle for internal transport powered by a hydrogen fuel cell and batteries with autonomous operation capacity (Figure 1). Its main features are rear-wheel drive, front-wheel steering, power $3.3 \mathrm{~kW}$, mass $690 \mathrm{~kg}$, top speed $32 \mathrm{~km} / \mathrm{h}$, length $2.66 \mathrm{~m}$, width 1.23 $\mathrm{m}$, height $1.70 \mathrm{~m}$, wheelbase $L=1.65 \mathrm{~m}$, height of the center of gravity $(G) h=0.50 \mathrm{~m}$, distance from $G$ to the front axle $L_{a}=1.10 \mathrm{~m}$, and distance from $G$ to the rear axle $L_{b}=0.55 \mathrm{~m}$.

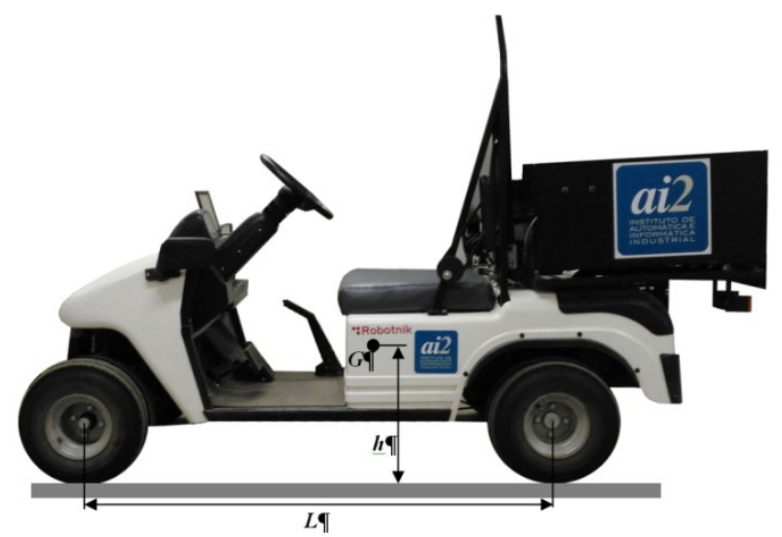

Figure 1. RBK car-like mobile robot

The model used is based on the well-known "bicycle model" (Figure 2), which is defined in more detail in [19]. It gives rise to the following simplifying assumptions:

- No roll and pitch motions.

- No side-load transfer.

- No aerodynamic effects.

- A plane model with three degrees of freedom and a restriction associated with the steering angle.

- The front wheels are simplified into one that will exert the force corresponding to both, and the same simplification applies to the rear wheels.

- The steering angle corresponds to that of the single front wheel of the model.

- Sideslip angles and their gradient are small. 


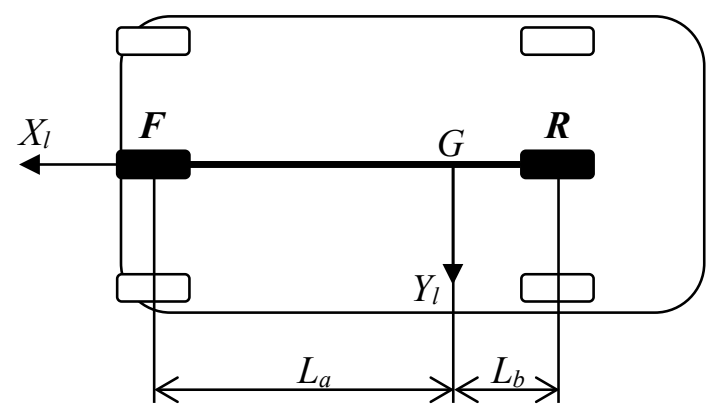

Figure 2. Bicycle model

\subsection{Robot kinematics}

A general trajectory, expressed in a polynomial form, as explained in section 3, is imposed on the center of gravity of the car-like robot (point $G$ in Figures 1 and 2). Thus, the kinematics of the center of gravity $G$ with respect to a global reference system is fully determined. It gives:

Position of $G$ :

$$
\begin{aligned}
& x_{G}=f_{x}(t) \\
& y_{G}=f_{y}(t)
\end{aligned}
$$

Velocity of G:

$$
\overrightarrow{V_{G}}=\dot{f}_{x}(t) \cdot \vec{\imath}+\dot{f}_{y}(t) \cdot \vec{\jmath}
$$

Considering the sideslip angle of the vehicle, $\beta$ small (see Figure 3 ) and $\dot{\beta}$ negligible, the angular velocity of the vehicle is obtained as:

$$
\vec{\omega}=\frac{\dot{f}_{x}(t) \cdot \ddot{f}_{y}(t)-\dot{f}_{y}(t) \cdot \ddot{f}_{x}(t)}{\left(\dot{f}_{x}(t)\right)^{2}+\left(\dot{f}_{y}(t)\right)^{2}} \cdot \vec{k}
$$

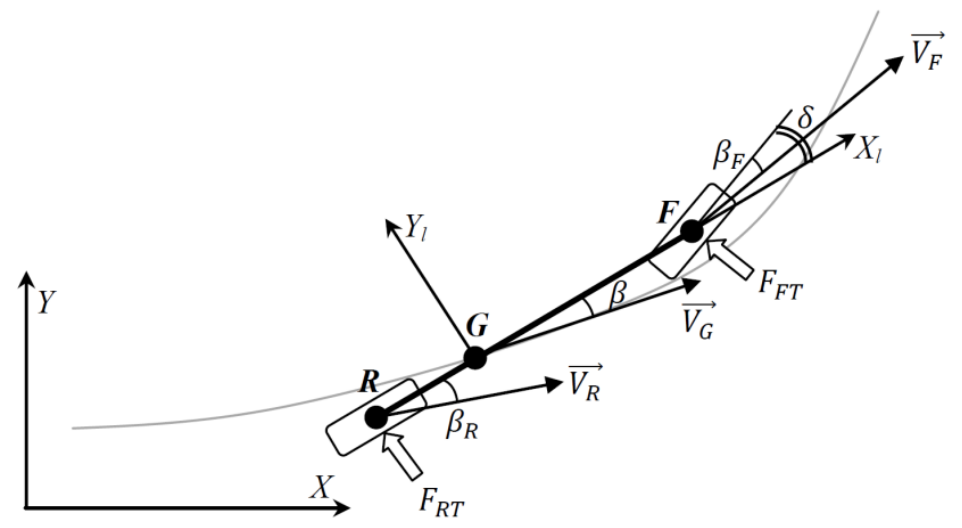

Figure 3. Kinematics and sideslip angles

Based on the previous kinematics, the rear sideslip angle $\beta_{R}$ is:

$$
\beta_{R}=\tan ^{-1} \frac{\left(\left|\overrightarrow{V_{G}}\right| \beta-L_{b} \omega\right)}{\left|\overrightarrow{V_{G}}\right|}
$$

Under the hypothesis of a small sideslip angle and considering trigonometric simplifications, it remains as:

$$
\beta_{R} \approx \beta-\frac{\left(\dot{f}_{x}(t) \cdot \ddot{f}_{y}(t)-\dot{f}_{y}(t) \cdot \ddot{f}_{x}(t)\right) L_{b}}{\left(\left(\dot{f}_{x}(t)\right)^{2}+\left(\dot{f}_{y}(t)\right)^{2}\right)^{3 / 2}}
$$


By proceeding analogously, the forward sideslip angle $\beta_{F}$ can be obtained:

$$
\beta_{F} \approx \delta-\beta-\frac{\left(\dot{f}_{x}(t) \cdot \ddot{f}_{y}(t)-\dot{f}_{y}(t) \cdot \ddot{f}_{x}(t)\right) L_{a}}{\left(\left(\dot{f}_{x}(t)\right)^{2}+\left(\dot{f}_{y}(t)\right)^{2}\right)^{3 / 2}}
$$

Taking $\theta$ as the orientation of the velocity $\vec{V}_{G}$ in the $X Y$ plane of the global reference system, the normal and tangential accelerations are:

$$
\begin{aligned}
& A_{G}^{n}=-\ddot{f}_{x}(t) \sin \theta+\ddot{f}_{y}(t) \cos \theta \\
& A_{G}^{t}=\ddot{f}_{x}(t) \cos \theta+\ddot{f}_{y}(t) \sin \theta
\end{aligned}
$$

In the local reference system that is linked to the vehicle (see Figure 3), the lateral acceleration of $G\left(\right.$ direction $\left.Y_{l}\right)$ is:

$$
A_{G}^{Y_{l}}=A_{G}^{n} \cos \beta-A_{G}^{t} \sin \beta
$$

Since the angle $\beta$ is small, this equation can be written as:

$$
A_{G}^{Y_{l}} \approx-\ddot{f}_{x}(t)(\sin \theta+\beta \cos \theta)+\ddot{f}_{y}(t)(\cos \theta-\beta \sin \theta)
$$

\subsection{Obtaining the sideslip and direction angles}

Under the small sideslip hypothesis, it is usual to consider the lateral behavior of the tires linearly (with $C_{T}$ stiffness), so as the front and rear tires are equal, the lateral forces are:

$$
\begin{aligned}
& F_{R T}=-C_{T} \beta_{R} \\
& F_{F T}=-C_{T} \beta_{F}
\end{aligned}
$$

with a direction normal to the rim and opposite to the sideslip (see Figure 3).

Setting the Newton-Euler equations for the lateral forces and the moments, the following expression is met:

$$
F_{R T}+F_{F T} \cos \delta=m A_{G}^{Y_{l}}
$$

where $\delta$ is the direction angle. Substituting (8) in the previous equation:

$$
-C_{T} \beta_{R}-C_{T} \beta_{F} \cos \delta=m A_{G}^{Y_{l}}
$$

The equation of moments is:

$$
F_{F T} \cos \delta L_{a}-F_{R T} L_{b}=I_{z} \dot{\omega}
$$

where $I_{Z}$ is the moment of inertia of the vehicle around an axis parallel to $Z$, passing through $G$, and operating:

$$
C_{T}\left(-L_{a} \beta_{F} \cos \delta+L_{b} \beta_{R}\right)=I_{z} \dot{\omega}
$$

Equations (4), (5), (9) and (10) are a system from which $\beta, \delta, \beta_{F}$ and $\beta_{R}$ can be obtained.

\section{Trajectories}

This section explains how the trajectory modeling is done and how the collision-free minimumtime trajectory is obtained.

\subsection{Modeling the trajectory}

The vehicle's center of gravity, $G$, must pass through a sequence of $m+1$ passing points joined by $m$ polynomial segments (according to expression (11)) that make up the trajectory, so that the following is met in section $j$ :

$$
\begin{aligned}
& x_{G j}=a_{x j}+b_{x j} t+d_{x j} t^{2}+e_{x j} t^{3} \\
& y_{G j}=a_{y j}+b_{y j} t+d_{y j} t^{2}+e_{y j} t^{3}
\end{aligned}
$$


$\forall t \in\left[0, t_{j}\right], t$ being the time variable associated with the robot's motion.

This sequence of $m$ polynomials should meet the following (8 (m-1)) continuity conditions:

- Continuity in positions. In each section, the initial and final position must be coincident with the passing point that defines it; $(4(m-1))$ equations are set.

- Continuity in velocities. The initial and final velocities of the trajectory must be zero; 4 equations are set. The initial velocity of each section must be equal to the final velocity of the previous one; $(2(m-2))$ equations are set.

- Continuity in accelerations. The initial acceleration of each section must be equal to the end of the previous one; $(2(m-2))$ equations are set.

Once the times associated with the different intervals forming the trajectory are known, a linear system of $(8(m-1))$ equations is available, which makes it possible to obtain the coefficients of the polynomials (11) that define the intervals so that the trajectory is fully determined.

\subsection{Minimum-time trajectory}

The aim is to obtain a trajectory of the type defined in section 3.1. that passes through a series of $m+1$ intermediate points and is of a minimum time. To achieve this, a problem of nonlinear optimization with non-linear constraints is posed, then the objective function and the constraints of the problem are specified.

\subsubsection{Objective function}

The trajectory will consist of $\mathrm{m}$ intervals or sections between the $m+1$ intermediate positions, where $t_{j}$ for $j=1 . . m$ are the times associated with the intervals that match the equations of type (11) and the associated conditions, so that the objective function is:

$$
f(t)=\sum_{j=1}^{m} t_{j}
$$

\subsubsection{Constraints}

i. The initial orientation of the robot $\theta_{i}$, corresponding to the initial position.

ii. The steering wheel angle does not exceed the specified value $\delta_{\max }$.

iii. The maximum speed of the vehicle cannot exceed $V_{\max }$.

iv. The driving force is limited by the torque curve of the engine.

v. The adhesion of the tires to the terrain is limited.

vi. The energy consumed by the vehicle is limited.

This is an optimization problem with nonlinear constraints, whose solution is obtained by the NLPQLP Quadratic Programming Algorithm with Distributed and Non-Monotone Line Search created and proposed by Professor Klaus Schittkowski. It should be considered that, in each iteration, the linear system associated with obtaining the coefficients of the equations of type (11) will be solved using the normalized time method (see [20]) so as not to penalize the computation times and, additionally, the derivatives of the constraints are obtained by finite differences.

\subsection{Obtaining the collision-free trajectory}

The problem is to obtain an efficient and collision-free trajectory for a mobile robot in an environment with static obstacles. A trajectory is called efficient when it is near the minimum time with a low computational cost and it matches the restrictions imposed on the robot, as defined in Section 3.2.2. Collision detection is specific for each type of standard obstacle, considering the mobile robot as a rectangular shape that is delimited by four segments. For circles, the distance from each segment to the center of the circle is calculated and if it exceeds 
the radius there is no collision. For polygons, it is verified that there is no intersection between the segments corresponding to the mobile robot and those of the obstacles.

The initial data are:

- Information about the robot that is needed for modeling it, as described in Section 2.

- Information about obstacles and their locations.

- Initial position $\left(x_{G i}, y_{G i}\right)$ and orientation $\theta i$, and final position $\left(x_{G f}, y_{G f}\right)$ of the mobile robot.

The iterative procedure to obtain the collision-free trajectory is:

a. Calculation of the minimum-time trajectory $T_{i}^{\text {min }}$ in a single interval that passes through the initial $\left(x_{G i}, y_{G i}\right)$ and final $\left(x_{G f}, y_{G f}\right)$ points.

b. Search for collisions.

c. For the selected path ( $T_{i}^{\text {min }}$ for the first iteration), the first configuration with collision $c_{c}$ is identified as the one previous to it $c_{a}$ (see Figure 4). This trajectory will be called the generator of the offspring that might be obtained in point e.

d. Generation of adjacent positions. If the current trajectory and its generator have not collided with the same obstacle, four adjacent positions are generated from $c_{a}\left(p_{a j} ; j=1, \ldots, 4\right.$ see figure 4) by choosing the positions that are far enough from any obstacle $p_{a k}(0 \leq \mathrm{k} \leq 4)$; if none exist $(k=0)$, a configuration in the previous trajectory $c_{a-1}$ is searched for and the algorithm works recursively until it finds a configuration that results in $(k \neq 0)$.

In the event that the current trajectory and its generator have collided with the same obstacle, it will only branch out in the same direction that gave rise to the current trajectory. This branching with memory makes it possible to reduce the computation time, avoiding the generation of trajectories that go through areas that have already been explored.

e. Generation of offspring trajectories. For each of the adjacent positions obtained at point $d$. an offspring trajectory $T_{k}^{\min }(0<k \leq 4)$ is obtained, which contains all the passing points previous to $c_{a}$, point $p_{a j}$ and the end point of the generating trajectory. In addition, the algorithm associates each generated trajectory with its generating trajectory, the branching direction and the obstacle that has originated the collision and given rise to this branch.

f. Selection of the trajectory. The trajectories generated at point e. are placed in a set of trajectories ordered by time $T S=\left\{T_{1}^{\min } \ldots T_{p}^{\min }\right\}$. The minimum-time trajectory within the set $T_{1}^{\text {min }}$ is selected, taken out of TS and also checked for collisions. If there are any collisions, the algorithm returns to point c. This process is repeated (iterating) until a solution $T_{1}^{\min }$ without a collision is reached.

g.

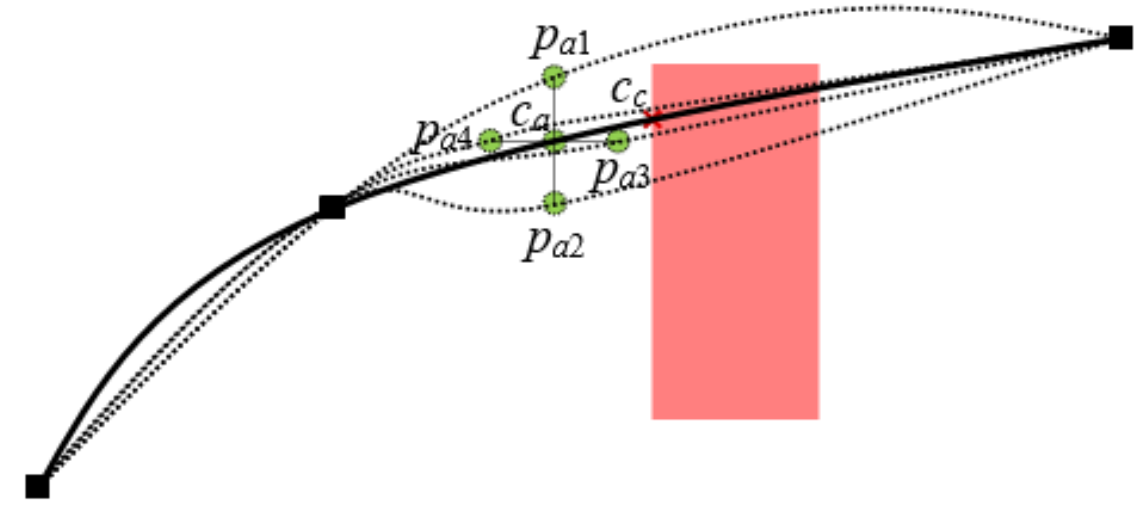

Figure 4. Generation of offspring trajectories (dotted lines) from the generating trajectory (solid line). 


\section{Case study}

From a given work environment with known obstacles, the autonomous vehicle should move from an initial configuration defined by a position and orientation to a final configuration defined by the vehicle's position (see Figure 5).

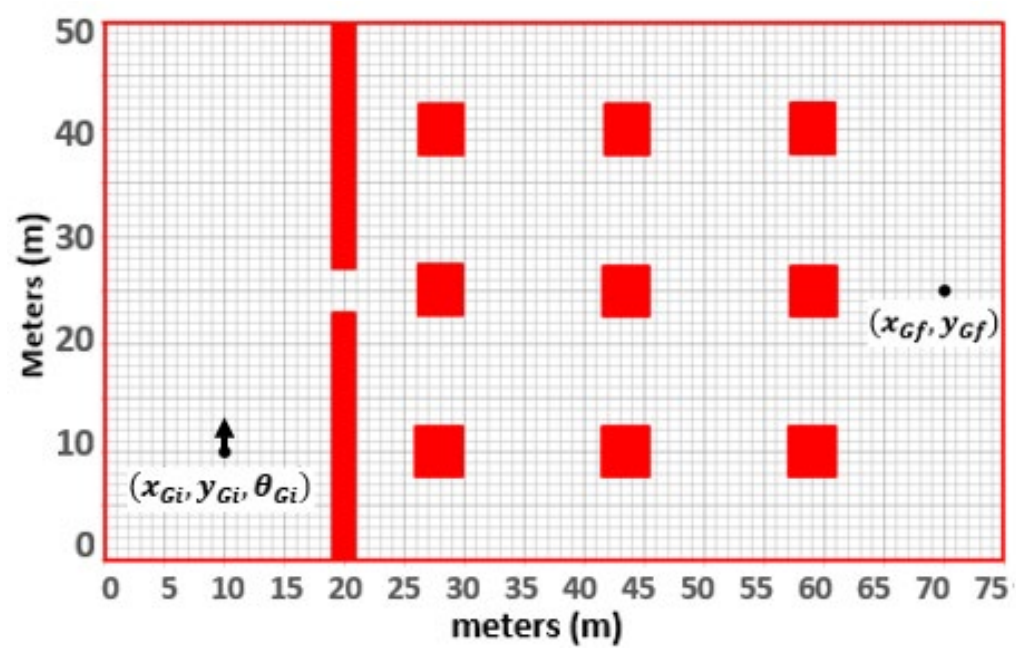

Figure 5. Work environment

Starting from the conditions established in Figure 5, the values of the constraint related to the energy consumption are applied. These values are obtained from a random sample, while keeping the characteristics of the vehicle and its interaction with the terrain that give rise to the rest of the constraints of the optimization problem unchanged. In this way, 101 trajectories have been obtained from the examples solved. To be more precise, the obtained trajectories have been generated keeping all the conditions constant except for the constraint of the energy consumed by the vehicle while in operation.

The energy consumed values are obtained using the Monte Carlo method applied to a variable subjected to uncertainty (that is, the energy consumed). To do this, a Gaussian statistical distribution is used with a mean $m=4800 \mathrm{~J}$ and a standard deviation of $m / 3$ and with random values of probability with uniform distribution. The criterion for selecting the normal distribution is based on the fact that random variables subjected to uncertainties are usually distributed normally when the number of samples is significantly high, as stated by the central limit theorem. In this way, it is possible to reproduce a set of real values of the energy consumed by the vehicle.

This Gaussian distribution can be seen in the following graphs. 


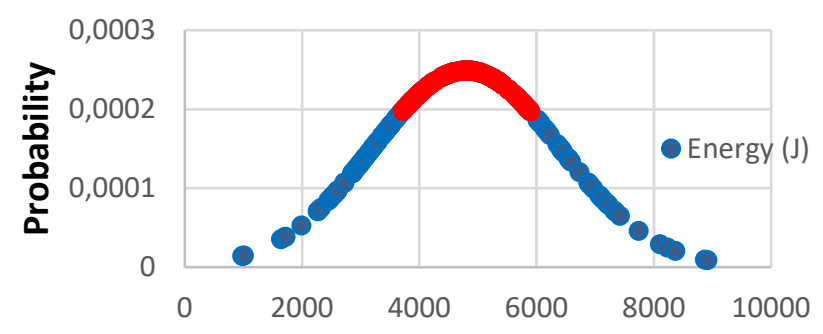

Gaussian Distribution of Energy Consumed (J)

Figure 6. Probability of the energy consumed, with a Gaussian distribution with mean value $m=4800 \mathrm{~J}$ and dispersion $m / 3$

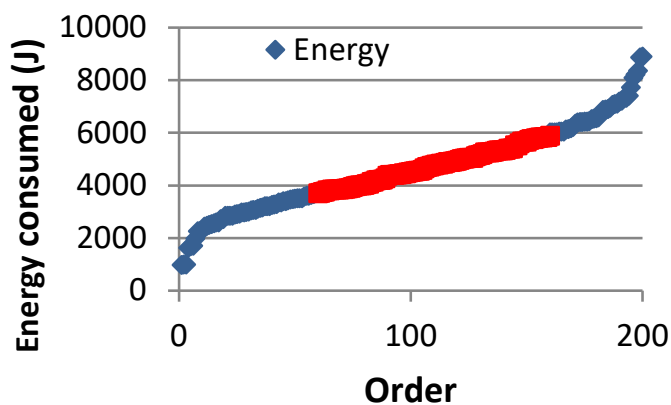

Figure 7. Ordered values of the energy consumed for the whole distribution and for the 101 selected cases

Of all the possible values of the energy consumed, those that appear in red have been selected (see Figure 6 and 7). They are values centered on the mean value $m$ with a dispersion $m / 3$. It is not appropriate to select all of them in order to draw conclusions regarding the behavior of the working parameters as a function of the energy consumed.

\section{Results and Findings}

From the 101 trajectories generated according to the conditions described in section 4 , the results obtained are shown below.

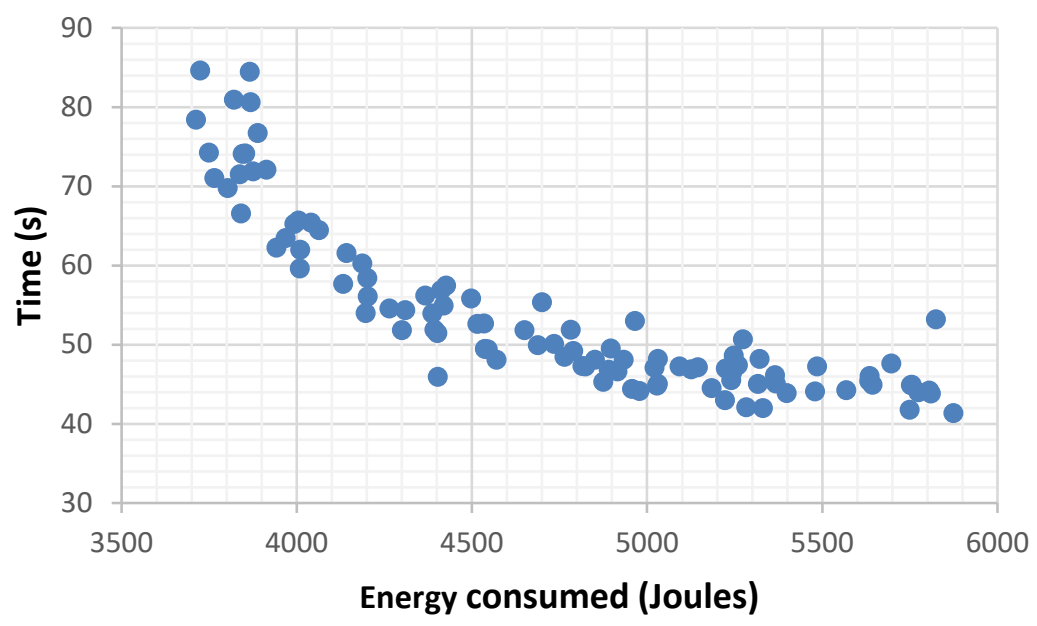

Figure 8. Energy consumed versus the time needed to run the trajectory

It can be seen in Figure 8 that for severe energy constraints (below 4000 Joules), high times are required and also the gradient is very high, while for high values of the constraint (from 5000 Joules) the trajectory time stabilizes. It can be observed how increasing the energy consumed does not improve the trajectory time above a certain threshold. 


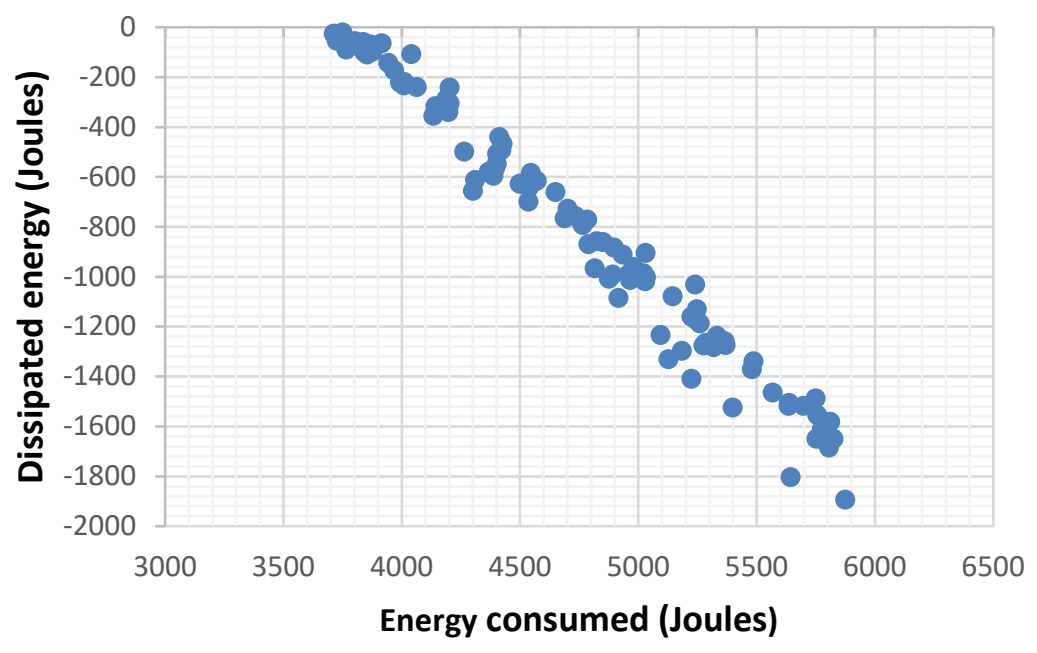

Figure 9. Dissipated energy versus energy consumed

Figure 9 shows the relationship between energy dissipated in the brakes and the energy consumed by the engine for different trajectories. It can be seen that there is a practically linear relationship: very high values of consumed energy imply a great dissipation associated with use of the brakes. Therefore, reducing trajectory times implies an increase in dissipated energy, which means there is a loss of energy.

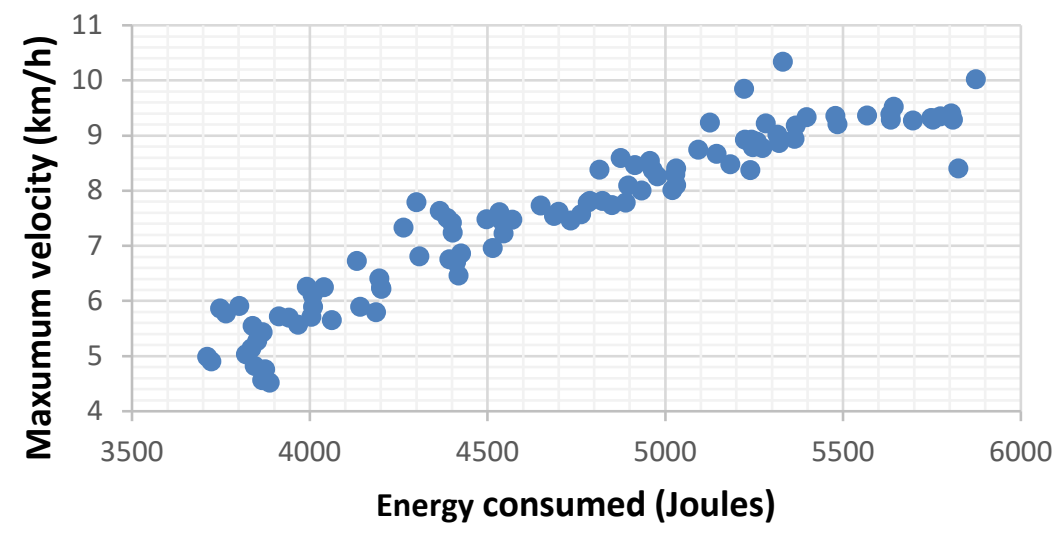

Figure 10. Maximum velocity reached versus the energy consumed in the trajectory

From the results shown in Figure 10, it can be seen that when the energy consumed is below 5400 Joules, the ratio between the maximum velocity reached by the vehicle and the energy consumed while running the trajectory is practically linear, and for higher values it remains stable. That is, large energy inputs do not lead to increases in speed. This behavior is associated with the limitations in the dynamic behavior of the vehicle, both for the limitations of the engine and for adhesion of the tires. 


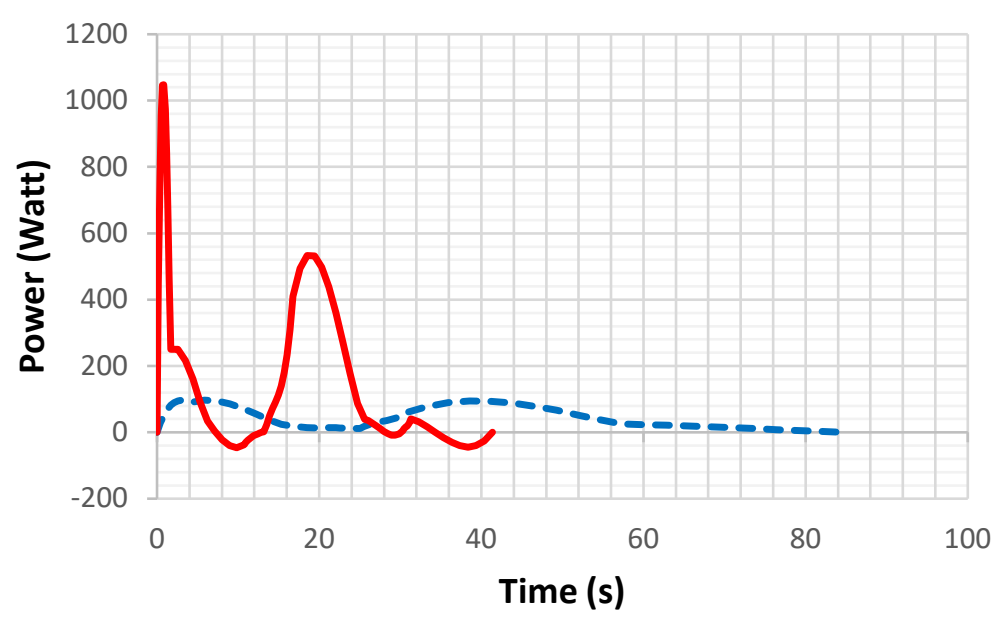

Figure 11. Power associated with the rear wheels for the fastest path (solid line) and for the slowest (dashed line), versus time

In the modeled vehicle, braking is allowed on all 4 wheels, with the front train tires braking more, while the traction is rear and thus the energy contribution is made only by the rear tires. It can be seen in Figure 11 that for the fastest path (solid line) the rear wheels brake (negative values in the power curve), leading to energy dissipation, while in the slowest case (dashed line) the power associated with the rear wheels is positive except for the last moments. In the case of the slowest trajectory the energy dissipation in the brakes is very low (24.34 Joules), so the energy is dissipated basically by rolling.

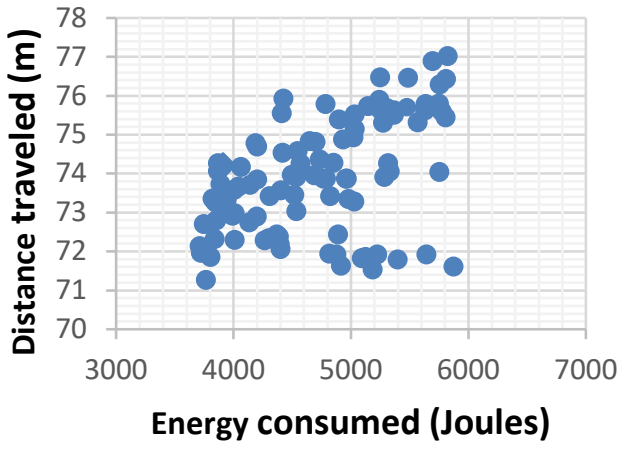

Figure 12. Distance traveled by the car-like robot against the energy consumed

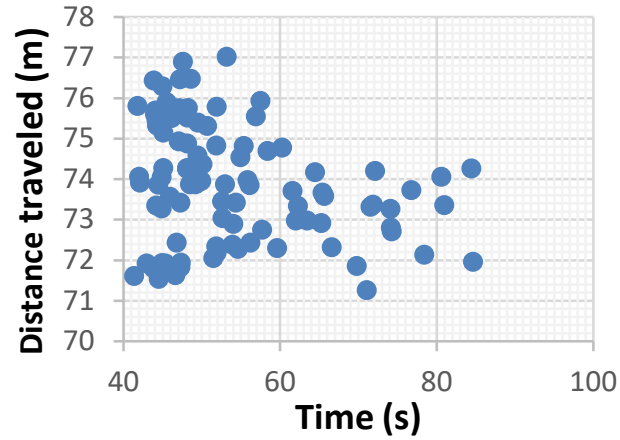

Figure 13. Distance traveled by the car-like robot versus the time taken to run the trajectory

The results shown in Figures 12 and 13 have a large dispersion, from which we can deduce that the distance traveled by the robot is influenced by factors other than the energy consumed. In addition, it should be noted that the difference between the longest and the shortest trajectory is less than 6 meters, which is $7.8 \%$ of the maximum distance. 


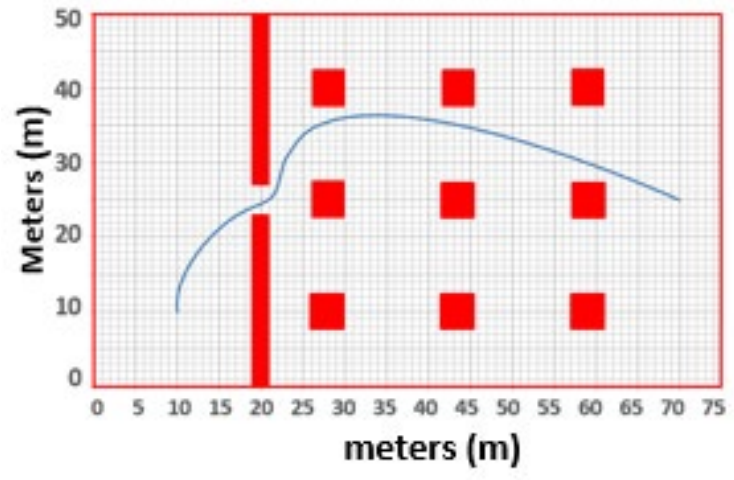

a

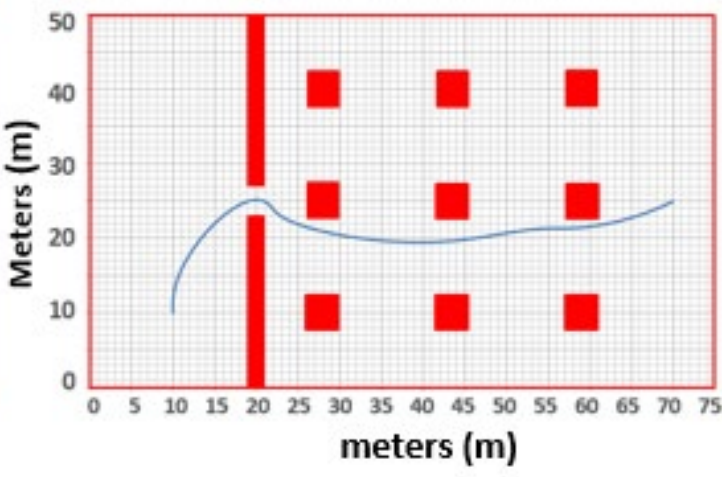

$\mathrm{b}$

Figure 14. a) Trajectory with 5824 Joules consumed; b) Trajectory with 5874 Joules consumed

Figure 14 shows two trajectories with very different paths but a similar energy consumption; in case a) the distance traveled is $77.03 \mathrm{~m}$ and in case b), $71.62 \mathrm{~m}$.

\section{Conclusions}

A main target of the paper is to look for patterns and relationships between the energy consumed by the car-like robot and the main operating parameters in order to optimize and reduce energy consumption.

From the 101 examples whose results are presented in section 5, a series of characteristics can be deduced regarding the behavior of the car-like robot as a function of the energy consumed, which makes it possible to establish a target energy consumption capable of improving the performance of the robot's work.

The following characteristics associated with the energy consumption of the robot can be highlighted:

- Very high energy consumption has little influence on the running times and the maximum velocities reached in the trajectories, but it does imply a significant increase in energy dissipation and it also means high expenses.

- Very low energy consumption has a great influence on the time needed to carry out the trajectories.

- The distances traveled by the robot have maximum differences of less than $8 \%$ of the maximum distance, but they are not dependent exclusively on the energy or the running time of the trajectory.

Working in a controlled industrial environment, mainly with regard to the adherence of the robot's tires that would allow it to maintain stable limits for the dynamic performance, a maximum profitability energy consumption restriction could be established by weighing the energy cost against the processing time.

\section{Acknowledgements}

\section{References}


[1] Marquez, C. et al., "Trajectory Generation for Wheeled Mobile Robots Via Bézier Polynomials," in IEEE Latin America Transactions, vol. 14, no. 11, pp. 4482-4490, Nov. 2016. doi: 10.1109/TLA.2016.7795818

[2] López, D.A., Nuevas aportaciones en algoritmos de planificación para la ejecución de maniobras en robots autónomos no holónomos. Tesis doctoral, 2011. URL: http://hdl.handle.net/10272/5501

[3] Choset, H., Lynch, K.M., Hutchinson, S., Kantor, G., Burgard, W., Kavraki, L.E., Thrun, S., Principles of Robot Motion: Theory, Algorithms, and Implementations. MIT Press, Boston (2005)

[4] Katrakazas, C., Quddus, M., Chen, W-H., \& Deka, L. (2015). Real-time motion planning methods for autonomous on-road driving: State-of-the-art and future research directions. Transportation Research, doi: 10.1016/ j.trc.2015.09.011

[5] Egerstedt, M. \& Hu, Xiaoming \& Stotsky, A. (1998). Control of a car-like robot using a dynamic model. IEEE International Conference on Robotics and Automation, 4. 3273 - 3278 vol.4. 10.1109/ROBOT.1998.680943.

[6] Jeon, J.H., Cowlagi, R.V., Peters, S.C., Karaman, S., Frazzoli, E., Tsiotras, P., \& Iagnemma, K. (2013). Optimal Motion Planning with the Half-Car Dynamical Model for Autonomous High-Speed Driving. American Control Conference (ACC)

[7] Ding, L., Deng, Z., Gao, H., Nagatani, K., \& Yoshida, K. (2011). Planetary rovers' wheelsoil interaction mechanics: new challenges and applications for wheeled mobile robots. Intelligent Service Robotics, 4(1), 17-38, DOI 10.1007/s11370-010-0080-5

[8] Valero, F., Rubio, F, Llopis-Albert, C. and Cuadrado, J.I., Influence of the Friction Coefficient on the Trajectory Performance for a Car-Like Robot, Mathematical Problems in Engineering, (2017), https://doi.org/10.1155/2017/4562647

[9] Kim, C., \& Kim, B. (2007). Minimum-Energy Rotational Trajectory Planning for Differential-Driven Wheeled Mobile Robots. In: Proceedings of 13th International Conference on Advanced Robotics (pp. 265-270).

[10] Tokekar, P., Karnad, N. \& Isler, Energy-optimal trajectory planning for car-like robots, Autonomous Robots (2014) 37-3: 279-300. https://doi.org/10.1007/s10514-014-9390-3

[11] Broderick, J.A., Tilbury, D.M. \& Atkins, E.M., Optimal coverage trajectories for a UGV with tradeoffs for energy and time, Autonomous Robots (2014) 36: 257. https://doi.org/10.1007/s10514-013-9348-x

[12] Wong, J. (2001). Theory of ground vehicles. New York: Wiley.

[13] Broderick, J., Tilbury, D., \& Atkins, E. (2012). Maximizing coverage for mobile robots while conserving energy. In Proceedings of the ASME 2012 IDETC/CIE.

[14] Oukacha, O. and Boizot, N. "Consumption minimisation for a car-like robot: Case study for a non-flat road profile," 2017 6th International Conference on Systems and Control (ICSC), Batna, 2017, pp. 334-341. doi: 10.1109/ICoSC.2017.7958672

[15] Salazar, M., Alessandretti, A., Aguiar, A.P., and Jones, C.N., "An energy efficient trajectory tracking controller for car-like vehicles using Model Predictive Control," 2015 54th IEEE Conference on Decision and Control (CDC), Osaka, 2015, pp. 3675-3680. doi: 10.1109/CDC.2015.7402789

[16] Liu, S. and Sun, D., "Minimizing energy consumption of wheeled mobile robots via optimal motion planning," Mechatronics, IEEE/ASME, Transactions on, vol. 19, no. 2, pp. 401-411, 2014

[17] Simba, K.R., Uchiyama, N., \& Sano, S. (2016). Real-time smooth trajectory generation for nonholonomic mobile robots using Bézier curves. Robotics and Computer-Integrated Manufacturing, 41, 31-42

[18] Rubio, F., Valero, F., Suner, J.L., \& Garrido, A. (2010). The Simultaneous Algorithm and the Best Interpolation Function for Trajectory Planning. Industrial Robot, 37 (5), 441-451.

[19] Valero, F., Rubio, F., Llopis-Albert, C. and Cuadrado, J.I., "Influence of the Friction Coefficient on the Trajectory Performance for a Car-Like Robot," Mathematical Problems in Engineering, vol. 2017, Article ID 4562647, 9 pages, 2017. doi:10.1155/2017/4562647 
[20] Suñer, J.L., Valero, F., Ródenas, J.J., and Besa, A., Comparación Entre Procedimientos de Solución de La Interpolación Por Funciones Splines Para La Planificación de Trayectorias de Robots Industriales, 2007.

[21] Luo, X., Li, S., Liu, S., \& Liu, G. (2018). An optimal trajectory planning method for path tracking of industrial robots. Robotica, 1-19. doi:10.1017/S0263574718001145 